\title{
Ba: Um Fator Determinante no Uso de Sistemas de Gestão do conhecimento
}

\author{
Nuno M. Sousa ${ }^{1}$, Carlos J. Costa ${ }^{1}$, Manuela Aparicio ${ }^{1,2}$ \\ nuno.m.sousa@icloud.com, carlos.costa@acm.org, manuela.aparicio@acm.org \\ ${ }^{1}$ Instituto Universitário de Lisboa (ISCTE-IUL) ISTAR-IUL, Lisboa, Portugal \\ ${ }^{2}$ Nova IMS, Universidade Nova de Lisboa, Portugal
}

DOI: 10.17013/risti.22.1-19

\begin{abstract}
Resumo: A gestão do conhecimento, no seio das organizações, constitui um modo muito importante na criação de dinâmicas competitivas para o mercado onde estas se inserem. Este artigo propõe um modelo estrutural de adoção, no qual " $B a$ ", designado aqui como a existência de um ambiente propício à partilha de conhecimento, é um fator determinante na adoção de sistemas organizacionais que se destinam à gestão do conhecimento. Este modelo foi validado através da aplicação de um inquérito em ambiente real de uma organização da área das telecomunicações. Verifica-se que “ $B a$ ”, por meio das tarefas tácitas organizacionais, é um fator significativo na perceção da facilidade de uso e por conseguinte fator determinante na adoção de sistemas de gestão do conhecimento.
\end{abstract}

Palavras-chave: $B a$, Partilha de conhecimento, Organização, Tarefa tácita, Sistemas de Gestão do Conhecimento, Cultura Organizacional.

\section{Ba: a Determinant Factor in Knowledge Management Systems' Use}

\begin{abstract}
Knowledge management is a very important way of creating competitive dynamics in the market. This paper proposes a structural adoption model, in which " $B a$ ", defined here, as the existence of a knowledge-sharing environment, is determinant in the adoption of organizational knowledge management systems (KMS). This model was validated through a survey within a real organizational context of a telecommunications company. Results showed that $\mathrm{Ba}$, through the tacit organizational tasks, is a significant factor in the perception of the ease of use and therefore a determinant factor in the adoption of KMS.
\end{abstract}

Keywords: Ba, Knowledge Sharing, Organization, Tacit Task, KMS, Knowledge Management Systems.

\section{Introdução}

Uma das principais preocupações que move as organizações atualmente, e em particular as empresas de telecomunicações, passa por definir a melhor forma de implementar os seus Sistemas de Informação (SI) tendo como foco não apenas a tecnologia mas 
também a informação e o conhecimento para suporte das vantagens de competitividade das organizações (Davenport \& Prusak, 1998; Hall, 2001). No que diz respeito à gestão do conhecimento é importante criar e implementar uma cultura que incentive a comunicação, a colaboração e a partilha da informação, definido por " $B a$ " (Nonaka \& Nishiguchi, 2001; Rubenstein \& Geisler, 2003). Foram efetuados diversos estudos relacionados com a Gestão do conhecimento ao longo dos anos, com o objetivo de validar o comportamento das pessoas em relação à partilha de conhecimento nas organizações e em que medida fatores como a influência social, a cultura organizacional e a tecnologia adotada, condicionam os colaboradores de uma organização (Galliers \& Leidner, 2014; Nonaka \& Nishiguchi, 2001; Van Baalen, Bloemhof-Ruwaard, \& Van Heck, 2005). Os estudos não demonstraram em que medida " $B a$ " pode ser determinante na adoção de sistemas KMS.

O trabalho de investigação aqui apresentado propõem um modelo estrutural de adoção, contribuindo para a operacionalização do conceito " $\mathrm{Ba}$ ", designado aqui como a existência de um ambiente propicio a partilha de conhecimento, assim como a identificação do seu impacto na gestão do conhecimento em contexto organizacional.

O presente artigo introduz uma revisão de literatura na segunda secção. Em seguida, é proposto na terceira secção um modelo. Finalmente, são apresentados os resultados e conclusões respetivamente na quarta e quinta secções.

\section{Revisão da Literatura}

\subsection{Conceito de Gestão do Conhecimento}

Na perspetiva epistemológica da apresentação de posse do conhecimento, esta realça os aspetos cognitivos, isto é, os aspetos de aquisição do conhecimento, sendo o conhecimento visto como fazendo parte exclusivamente da mente humana, a capacidade mental (ou cognitiva), onde o conhecimento tácito como o conhecimento explícito se complementam, numa dinâmica que caracteriza a natureza humana, através da sua criatividade e imaginação, possibilitando a criação de novo conhecimento e melhorar assim a eficácia e eficiência no local de trabalho (Martins, 2010; Newell et al., 2009). Na verdade, uma organização não é uma simples máquina de processamento de informação, mas sim uma entidade que cria conhecimento através da ação e interação das pessoas que a compõem, sendo imperativo fomentar uma dinâmica que permita o surgimento de novas ideias. Desta forma, a gestão do conhecimento é nada mais do que a gestão dinâmica do próprio processo de criação de conhecimento (Nonaka \& Nishiguchi, 2001; Nonaka, Toyama, \& Konno, 2000). Podemos afirmar, no âmbito deste artigo, que a gestão do conhecimento trata essencialmente do conhecimento existente, reportando a um conjunto de táticas, com o intuito de promover a implementação de técnicas e metodologias, orientadas por uma estratégia organizacional, sustentada numa cultura transparente, aberta e aceite no contexto social organizacional, com o objetivo de promover um ambiente propício à partilha de conhecimento entre colaboradores e stakeholders, conduzindo assim a uma melhoria continua dos serviços prestados pela organização aos seus clientes (Chaffey \& Wood, 2005; Maier, 2007; Maier \& Hädrich, 2011; Martins, 2010; Xavier, Oliveira, \& Teixeira, 2012). 


\subsection{A Cultura Organizacional na Gestão do Conhecimento}

A cultura organizacional é um fator importante na definição de uma gestão do conhecimento eficiente. Davenport et al. (1998), abordam o conceito de "KnowledgeFriendly Culture" como sendo um dos mais importantes fatores para o sucesso dos projetos nas organizações, bem como na aprendizagem mediada por sistemas de informação (Aparicio, Bacao \& Oliveira, 2016), sendo também um dos mais difíceis de criar caso não exista de todo na organização. Na gestão do conhecimento, na perspetiva das organizações, nomeadamente na área das Tecnologias da Informação (TI), não consideram como foco principal a forma como a informação é disponibilizada às pessoas, mas sim a necessidade de desenvolver um ambiente propício à partilha de conhecimento, porque não são os sistemas que melhoram o desempenho organizacional ou criam valor de negócio, mas sim as pessoas que trabalham para a organização (Junnarkar \& Brown, 1997; Markus \& Keil, 1994). No contexto da partilha de conhecimento, pré-condições culturais como a "abertura", o "clima de comunicação" e o "respeito mútuo", sendo este último definido pela confiança, compreensão e apreço mútuo, são considerados como tendo uma influência positiva no desenvolvimento do conhecimento, na partilha e na avaliação crítica da necessidade de conhecimento e disponibilidade desse conhecimento. Assim sendo, certos ambientes são mais propícios para a partilha de conhecimento quando a cultura corporativa encoraja à inovação e incute a responsabilidade da partilha de conhecimento entre colaboradores, promova comunidades que assumem a partilha de conhecimento como uma iniciativa, e ainda, o incentivo à experimentação dos Knowledge Management Systems (KMS) disponibilizados pelas organizações (Gomes \& Romão, 2012; Hall, 2001; van den Hooff et al., 2003).

\subsection{Ba: Ambiente Propício à Partilha de Conhecimento}

Considera-se, no contexto do presente artigo, que um ambiente propício à partilha de conhecimento é formulado pela interceção de quatro variáveis influenciadoras e distintas (Castellani et al., 1998; Fishbein \& Ajzen, 1975; Fleury, 1997; Gold et al., 2001; Venkatesh \& Davis, 2000). A primeira variável consiste em o "Individuo", na visão do utilizador das soluções KMS e na disposição de partilhar o seu conhecimento. A segunda variável é observada a "Organização", no que se refere à cultura organizacional instituída, no sentido de abertura à partilha e aprendizagem do conhecimento. A terceira variável consiste no ambiente "Social", enquanto influenciador do comportamento do colaborador inserido num registo de convivência diária com os seus pares/colegas de trabalho. Como última variável, o ambiente "Virtual", referenciando a componente que compõe a infraestrutura tecnológica utilizada para expressar, registar e partilhar os diferentes tipos de conhecimento que compõem as diferentes componentes do negócio. Alguns autores referem-se a " $B a$ " como sendo um lugar de interação no mundo virtual, onde é combinado o novo conhecimento explícito com a informação existente (Nonaka \& Nishiguchi, 2001). $\mathrm{O}$ ambiente propício à partilha do conhecimento, proposto neste artigo, tem como referência " $B a$ ", já que " $B a$ " é a relação de espaço-tempo, ou seja, determina o lugar e o tempo onde a informação é interpretada para tornar-se conhecimento, sendo esta parte da função mental do indivíduo (Leonard \& Sensiper, 1998; Nonaka \& Konno, 1998; Polanyi, 1958; Wilson, 2002), tal como apresentado na Figura 1. 


\begin{tabular}{|c|c|c|c|}
\hline \multicolumn{4}{|c|}{ Ambiente Propício à Partilha de Conhecimento (“ $B a$ ") } \\
\hline$\downarrow$ & $\downarrow$ & $\downarrow$ & $\downarrow$ \\
\hline $\begin{array}{l}\text { Fatores Individuais } \\
\text { (FI) }\end{array}$ & $\begin{array}{c}\text { Favorecimento da } \\
\text { Cultura Organiza- } \\
\text { cional (FCO) }\end{array}$ & $\begin{array}{l}\text { Influência Social } \\
\text { (IS) }\end{array}$ & $\begin{array}{c}\text { Infraestrutura } \\
\text { Tecnológica KM } \\
\text { (TKMI) }\end{array}$ \\
\hline $\begin{array}{l}\text { Prazer em ajudar os } \\
\text { outros; Conhecimento } \\
\text { de auto-eficácia }\end{array}$ & $\begin{array}{l}\text { Cultura } \\
\text { Organizacional }\end{array}$ & Normas Subjetivas & $\begin{array}{l}\text { Infraestruturas } \\
\text { Tecnológicas }\end{array}$ \\
\hline Individuo & Organização & Social & Virtual \\
\hline
\end{tabular}

Figura 1 - Ambiente Propício à Partilha de Conhecimento ("Ba”) - "fonte própria"

Desta forma, pode-se considerar o "Ambiente Propício à Partilha do Conhecimento ("Ba")», como um agregador de quatro dimensões apuradas na pesquisa, "Fatores Individuais (FI)" (Lin, 2007), "Favorecimento da Cultura Organizacional (FCO)" (Rosenberg, 2000; Tyan, 2004; Van Dam \& Van Dam, 2004; Vasconcellos \& Fleury, 2008), "Influência Social (IS)" (Venkatesh \& Davis, 2000; Venkatesh, Morris, Davis, \& Davis, 2003) e a "Technological KM Infrastructure (TKMI)" (Gold et al., 2001; Lousã \& Sarmento, 2016).

\subsection{KMS: Knowledge Management Systems}

O conhecimento adquirido pelas pessoas e o produto resultado da aplicação desse conhecimento, assume o fator mais relevante na gestão do conhecimento (Schütt, 2003). É este o conhecimento que é considerado importante para as organizações, na medida que permite a resolução de problemas, evolução de projetos, elaboração de um produto ou serviço, entre outros, definido como "knowledge workers", sendo que esse conhecimento vital não pode residir apenas na mente dos indivíduos, deve ser externalizado, isto é, deve ser explicitado com o apoio de soluções colaborativas avançadas. Numa empresa de telecomunicações, que opera num ambiente de constante mudança tecnológica e num cenário altamente competitivo, a aplicação de "dynamic capabilities frameworks", permitem uma análise dos recursos e métodos como forma de criação de riqueza. Estas medidas estratégicas permitem alcançar a sustentabilidade e vantagem competitiva, dependendo em grande parte do aperfeiçoamento dos processos tecnológicos, organizacionais e de gestão, que permitem um crescimento continuo e da valorização do capital humano (conhecimento, habilidades e experiência) (Helfat \& Peteraf, 2003; Teece et al., 1997). Alguns dos termos adotados, resultado das diferentes abordagens no âmbito da grande diversidade de estudos efetuados, tais como: "knowledge-based information system", "knowledge management software", "knowledge portal", entre outros, resultam também em abordagens tecnológicas corporativas, isto é, as organizações investem na criação de soluções tecnológicas como plataformas abrangentes que pretendem colmatar o défice organizacional, fundamentando-se num leque de conceitos ontologicamente baseadas em Knowledge Management (KM), com o objetivo final de fomentar uma memória organizacional eficiente onde a partilha de informação é a chave principal para o sucesso organizacional (Maier, 2007). Podemos considerar assim, no âmbito deste artigo, que KMS, define-se como sendo um conjunto de sistemas de Information and Communication Technology 
(ICT) colaborativos, que fomentam a partilha de conhecimento, através da utilização de serviços avançados, construídos de forma contextualizada e integrada, sustentada numa ontologia, que permite a ação de compartilhar através da aplicação de um vocabulário comum, personalizada e adaptada a uma rede de participantes que operam em comunidade, com o objetivo de alcançar a sustentabilidade e vantagem competitiva, através da valorização do capital humano (Helfat \& Peteraf, 2003; Maier, 2007; Maier \& Hädrich, 2011; Noy \& McGuinness, 2001).

\section{Proposta de Modelo}

Compreender a aceitação e o uso de uma determinada TI é um dos principais objetivos dos estudos inerentes aos SI, sendo que deve ser fácil de utilizar, confiável, atendendo a opinião e necessidades reais do utilizador final (Doll \& Torkzadeh, 1988; Pedrosa, Costa, \& Laureano, 2015; Venkatesh, Davis, \& Morris, 2007). O estudo aplica uma metodologia quantitativa, sendo que o modelo aqui proposto está sustentado na teoria da adoção de sistemas de informação, com o objetivo de avaliar o uso efetivo e possibilitar uma explicação dos fatores determinantes da utilização dos sistemas (Davis et al., 1989; Venkatesh et al., 2003).

\subsection{Dimensões}

O modelo proposto baseia-se na teoria da gestão do conhecimento e na teoria da adoção de SI. Na Tabela 1, apresentam-se as dimensões, que segundo a literatura, poderão ser fatores determinantes na partilha e adoção de sistemas de gestão do conhecimento. As subsecções seguintes apresentam cada uma dessas dimensões:

\subsection{Proposta das Hipóteses}

No levantamento das hipóteses, em relação às dimensões internas relacionadas diretamente com o modelo Technology Acceptance Model (TAM), adotado no estudo, as hipóteses são demonstradas pelos estudos de Davis (1986).

A dimensão Facilidade de Uso Percebida (FUP) tem sido demonstrado na literatura como sendo importante na aceitação de tecnologia, representando o grau ao qual uma pessoa acredita que usando uma tecnologia específica seria livre de esforço (Davis et al., 1989). As Tarefas Tácitas (TT), definem as ações que são realizadas pelos indivíduos, quando geram conhecimento, com a transformação da informação através de inputs e outputs num sistema tecnológico (Goodhue \& Thompson, 1995). Estudos efetuados na área da gestão do conhecimento relataram a dificuldade no uso da tecnologia enquanto fronteira a transpor pelo indivíduo que tem de executar as suas tarefas, com determinado esforço e tempo despendido no uso dos sistemas KMS (Goodman \& Darr, 1998). Para além disso, Goodman \& Darr (1998), afirmam que a complexidade do problema pode inibir a mudança, neste caso o uso dos sistemas KMS, assim como a diferença entre o conhecimento explícito na organização e o conhecimento tácito do colaborador. Pretendese assim, compreender com a hipótese H1, em que medida a TT exercida pelo individuo no uso de um sistema KMS influencia positivamente a FUP desse mesmo sistema KMS.

H1: A Tarefa Tácita (TT) terá um efeito positivo significativo sobre a Facilidade de Uso Percebida (FUP) 


\begin{tabular}{|c|c|c|}
\hline Dimensão & Descrição & Autores \\
\hline Tarefa Tácita & $\begin{array}{l}\text { O equilíbrio entre o conhecimento tácito e o } \\
\text { conhecimento explícito. }\end{array}$ & $\begin{array}{l}\text { Goodhue \& Thompson, (1995) } \\
\text { Jarvenpaa \& Staples, (2000) }\end{array}$ \\
\hline $\begin{array}{l}\text { Interdependência de } \\
\text { Tarefa }\end{array}$ & $\begin{array}{l}\text { A existência de partilha de saberes que } \\
\text { permitem concretizar a tarefa. }\end{array}$ & $\begin{array}{l}\text { Goodhue \& Thompson, (1995) } \\
\text { Kankanhalli, Tan, \& Wei, (2005) }\end{array}$ \\
\hline $\begin{array}{l}\text { Cultura } \\
\text { Organizacional }\end{array}$ & $\begin{array}{l}\text { Assume o papel mais importante no contexto } \\
\text { social, sendo que os valores influenciam } \\
\text { direta ou indiretamente o uso de ferramentas } \\
\text { KM. }\end{array}$ & $\begin{array}{l}\text { Davis Jr, (1986); Fleury, (1997); } \\
\text { Alavi, Kayworth, \& Leidner, } \\
\text { (2005); Vasconcellos \& Fleury, } \\
\text { (2008) }\end{array}$ \\
\hline Influência Social & $\begin{array}{l}\text { Define o grau pelo qual um indivíduo } \\
\text { percebe o quanto é importante que os outros } \\
\text { acreditam que devem utilizar o sistema }\end{array}$ & $\begin{array}{l}\text { Venkatesh et al., (2003); } \\
\text { Venkatesh \& Davis, (2000) }\end{array}$ \\
\hline Fatores Individuais & $\begin{array}{l}\text { Fator referente ao comportamento do } \\
\text { individuo influenciado pelas suas crenças, } \\
\text { valores, motivações e experiência, enquanto } \\
\text { colaborador na organização. }\end{array}$ & $\begin{array}{l}\text { Lin, (2007); M McLure Wasko \& } \\
\text { Faraj, (2000) }\end{array}$ \\
\hline $\begin{array}{l}\text { Technological KM } \\
\text { Infrastructure }\end{array}$ & $\begin{array}{l}\text { Define a dimensão tecnológica como a } \\
\text { geração de oportunidade na realização das } \\
\text { tarefas. }\end{array}$ & $\begin{array}{l}\text { (Gold et al., 2001; Lousã \& } \\
\text { Sarmento, 2016) }\end{array}$ \\
\hline Utilidade Percebida & $\begin{array}{l}\text { Define o grau e que uma pessoa acredita que } \\
\text { usar um sistema em particular aumenta o } \\
\text { desempenho do seu trabalho }\end{array}$ & $\begin{array}{l}\text { (Davis et al., 1989; Venkatesh \& } \\
\text { Davis, 2000) }\end{array}$ \\
\hline $\begin{array}{l}\text { Facilidade de Uso } \\
\text { Percebida }\end{array}$ & $\begin{array}{l}\text { Define o grau a que uma pessoa acredita que } \\
\text { usando um determinado sistema estaria livre } \\
\text { do esforço }\end{array}$ & $\begin{array}{l}\text { (Davis et al., 1989; Venkatesh \& } \\
\text { Davis, 2000) }\end{array}$ \\
\hline $\begin{array}{l}\text { Intenção } \\
\text { Comportamental com } \\
\text { relação ao uso }\end{array}$ & $\begin{array}{l}\text { O grau de afeto avaliativo que um indivíduo } \\
\text { associa quando usa o sistema no seu trabalho }\end{array}$ & $\begin{array}{l}\text { (Davis et al., 1989; Venkatesh \& } \\
\text { Davis, 200o) }\end{array}$ \\
\hline $\begin{array}{l}\text { Uso efetivo do } \\
\text { sistema }\end{array}$ & $\begin{array}{l}\text { Resposta comportamental da intenção do } \\
\text { indivíduo em utilizar o sistema }\end{array}$ & $\begin{array}{l}\text { (Davis et al., 1989; Venkatesh \& } \\
\text { Davis, 2000) }\end{array}$ \\
\hline
\end{tabular}

Tabela 1 - Dimensões do modelo de adoção de KMS

Ainvestigação de Davis (1986), concentrou-senestas duas dimensões, Utilidade Percebida (UP) e a Facilidade de Uso Percebida (FUP), teorizadas para fundamentar a utilização do sistema. Davis, Bagozzi e Warshaw (1989) e Costa, Ferreira, Bento e Aparicio (2016), demonstraram que estas duas dimensões são estatisticamente distintas, apresentando medidas que indicam o uso do sistema em análise, onde FUP terá um efeito direto sobre UP, na medida em que o aumento de FUP contribui para melhorar o desempenho da tarefa, onde UP é influenciada pela FUP e por variáveis externas, desta forma propõe-se a hipótese H2, onde FUP terá um efeito positivo e significativo sobre UP:

H2: A Facilidade de Uso Percebida (FUP) tem um efeito positivo significativo sobre a Utilidade Percebida (UP)

A Intenção Comportamental do Uso (BI) é atualmente o melhor preditor para o uso de sistemas, já que é determinado pela atitude comportamental no uso dos sistemas, esta atitude, por sua vez é determinada pela UP e pela FUP (Davis \& Venkatesh, 1996; 
Davis Jr, 1986). Tanto o modelo original de Davis (1986) como pesquisas posteriores demonstraram que o efeito de UP sobre BI era apenas parcialmente medida pela atitude em relação ao uso, sendo explicado pelo facto de que no ambiente de trabalho as pessoas podem utilizar uma determinada tecnologia independentemente de terem ou não uma atitude positiva para o uso dessa tecnologia, desde que essa tecnologia permita o alcance da produtividade no seu trabalho (Davis \& Venkatesh, 1996; Costa et. al., 2016). Desta forma são propostas as hipóteses $\mathrm{H}_{3}, \mathrm{H}_{4}$ e $\mathrm{H}_{5}$ com que se pretende determinar o efeito positivo e significativo exercido.

H3: A Facilidade de Uso Percebida (FUP) terá um efeito positivo significativo sobre a Intenção Comportamental do Uso (BI)

H4: A Utilidade Percebida (UP) terá um efeito positivo significativo sobre a Intenção Comportamental do Uso (BI)

H5: A Intenção Comportamental do Uso (BI) terá um efeito positivo significativo sobre a Uso Efetivo (USE)

O conceito de " $B a$ ", enquanto dimensão enraizada na filosofia Japonesa, é apresentado neste estudo como conceito abstrato que explica o espaço ou o Ambiente Propícioà Partilha de Conhecimento (Alavi et al., 2005; Nonaka \& Konno, 1998). O desenvolvimento de um ambiente organizacional que permita a partilha do conhecimento de forma natural, ou seja, um ambiente propício à partilha de conhecimento, assume o papel de maior relevo no processo (Junnarkar \& Brown, 1997). No entanto, as pessoas podem sentir alguma relutância quando são convidadas a participar na partilha de conhecimento, e como tal, no uso de sistemas dedicados ao processo quando a confiança é parte do problema (Rubenstein \& Geisler, 2003; Ruppel \& Harrington, 2000). Abordando a framework SECI, de Nonaka \& Takeuchi (1995), o enquadramento de " $B a$ " é visível nos quatro quadrantes do modelo SECI, sendo expressivo neste contexto nos quadrantes "externalização" e "internalização" respetivamente: "Dialoguing Ba", o espaço e momento onde o conhecimento tácito é transferido e documentado na forma explícita; e "Exercising Ba" como sendo o espaço e momento onde o conhecimento explícito é convertido em conhecimento tácito (Nonaka, 1994; Nonaka \& Konno, 1998). As tarefas tácitas são enquadradas no contexto do indivíduo que usa um determinado sistema KMS, acima de tudo uma tarefa cognitiva que se funde com um contexto social, e como tal não são facilmente descartáveis como o uso de uma ferramenta física. Estas tarefas enquadram-se no "saber" mas dependem também do "fazer", enquanto no uso da tecnologia, sendo que estes dois aspetos são inseparáveis, denominando-se por "knowledge how" e "knowledge what", tendo em conta que o conhecimento tácito suporta dois aspetos relevantes: o "aspeto técnico" e "aspeto cognitivo" (Polanyi, 1958; Ryle, 2009). Podemos considerar para a hipótese H6, como relevante, para entender em que medida " $B a$ " exerce um efeito positivo e significativo na Tarefa Tácita do indivíduo quando do uso de KMS.

H6: O Ambiente Propício à Partilha do Conhecimento ("Ba") tem um efeito positivo significativo sobre a Tarefa Tácita (TT)

Alguns estudos revelaram que uma cultura organizacional não consolidada, com falha em alguns dos mecanismos relevantes como o clima de comunicação e a abertura, pode repercutir resultados desastrosos no uso efetivo das soluções KMS que as organizações 
disponibilizam com o objetivo de fomentar uma política de partilhar de conhecimento na organização (Rubenstein \& Geisler, 2003; Ruppel \& Harrington, 2000). As plataformas ICT colaborativas e integradas que permitam a partilha de conhecimento, assumindo uma base ontológica relacionada com o ato de compartilhar, são fundamentais no uso da tecnologia por parte dos participantes, dessa forma, é importante conhecer em que medida as infraestruturas tecnológicas afetam o processo de partilha de conhecimento (Aparicio et al., 2016), já que a tecnologia é um elemento crucial na dimensão estrutural para mobilizar o capital social no processo de criação de novo conhecimento na organização. Assim, a tecnologia é enquadrada na componente virtual, como parte de "Ba", como fator condicionador nas tarefas do indivíduo, tendo em conta as chaves que a caracterizam: a tecnologia, a cultura e a estrutura organizacional, no ambiente como sendo em parte o local da organização, onde é executada a tarefa pelo individuo quando do uso dos sistemas KMS (Alavi et al., 2005; Fleury, 1997; Gold et al., 2001; Maier \& Hädrich, 2011; Nonaka \& Konno, 1998; Noy \& McGuinness, 2001).

H7: O Ambiente Propício à Partilha do Conhecimento (“Ba") é uma dimensão de segunda ordem de tipo refletivo-refletivo, que se reflete nas seguintes dimensões: Favorecimento da Cultura Organizacional (FCO), Influência Social (IS) e Infraestrutura Tecnológica $K M$ (TKMI)

\section{Validação do Modelo de Adoção de Sistemas de Gestão do Conhecimento}

\subsection{Definição da amostra}

O estudo ocorreu durante o último trimestre de 2016, e foi conduzido em ambiente real de uma organização que opera na área das telecomunicações, com a participação dos profissionais das 16 áreas que compõem a direção de suporte e qualidade, sendo identificados para o estudo 3 sistemas de gestão do conhecimento atualmente em utilização: (1) Confluence (Atlassian Software Systems Pty Ltd, 2013); (2) JIRA (Atlassian Software Systems, 2012); (3) SharePoint (Microsoft Coorporation, 2010). As soluções Content Management System (CMS), apresentadas no estudo baseiam-se numa arquitetura centralizada "Server-based", mas sem camada integradora com outros sistemas ou bases de dados que compõem a infraestrutura dos SI da organização. A plataforma da Atlassian: JIRA e Confluence, contem uma vertente "peer-to-peer" (p2p), no entanto sem componentes de arquitetura "super-peer" configuradas. A iniciativa KM apresenta uma característica estratégica personalizada, ao encontro de numa filosofia de design organizacional descentralizada, em que o conteúdo resume o registo de documentos individuais, ideias, procedimentos, resultado da experiência no trabalho do dia-a-dia, tendo como base uma cultura organizacional aberta e de confiança intrínseca nos colaboradores da organização (Maier \& Hädrich, 2011).

\subsection{Caracterização da Amostra}

Os dados recolhidos através do recurso a um inquérito (consultar Apêndice A) difundido internamente, foram tratados com recurso ao método SEM/PLS, o método dos mínimos quadrados parciais. Os dados foram tratados estatisticamente com recurso ao programa Smart PLS. A amostra foi considerada adequada ao modelo (Cohen, 1992; 
Hair et al., 2014)"issued”:\{“date-parts":[["1992”]]\}\}\},\{“id”:184,"uris":[“http://zotero. org/users/3129792/items/A3DPDHE3"],"uri":["http://zotero.org/users/3129792/ items/A3DPDHE3"],"itemData":\{“id":184,"type":"book","title":"A primer on partial least squares structural equation modeling (PLS-SEM. A taxa de resposta foi de 39\% do universo formado por colaboradores internos e externos, da direção de suporte dos SI da empresa de telecomunicações. Com uma média de idade de aproximadamente 37 anos, foi apurado o total de 253 respostas ao inquérito, representativo das 3 soluções CMS em uso. A distribuição por cada solução CMS é visível na Tabela 2.

\begin{tabular}{lll}
\hline Soluções CMS & Frequência absoluta & Frequência relativa p/sistema \\
\hline Confluence & 94 & $37,15 \%$ \\
\hline JIRA & 76 & $30,04 \%$ \\
\hline SharePoint & 83 & $32,81 \%$ \\
\hline TOTAL & $\mathbf{2 5 3}$ & $\mathbf{1 0 0 \%}$ \\
\hline
\end{tabular}

Tabela 2 - Distribuição por Plataforma CMS

Em resposta a uma afirmação no inquérito, a maioria dos inquiridos (90\%) expressa vontade em utilizar apenas uma única plataforma KMS, que centralize a informação a registar e consultar, em vez de várias.

\subsection{Resultados}

Na avaliação da fiabilidade dos resultados obtidos, como apresentado na Tabela 3, referente aos Critérios de Qualidade, as variáveis latentes apresentam um Alpha de Cronbach superior a 0.85 , apresentando assim uma Fiabilidade de Consistência Interna (Hair et al., 2014). A Variância Média Extraída (AVE), tendo em conta os "loadings" superiores a 0.708 , permitiram assim analisar a Validade Convergente. Os valores AVE apresentamse superiores a 0.5 , para a dimensão refletivo-refletivo (segunda ordem) "Ba" e valores superiores a 0.7 para as restantes dimensões reflexivas. A Fiabilidade Composta Interna deve ser superior a 0.708 , embora na realização de pesquisa exploratória, 0.60 a 0.70 é considerado aceitável. Pode-se considerar a possibilidade do Alfa de Cronbach como sendo uma medida conservadora da Fiabilidade Consistência Interna (Hair et al., 2014).

$\mathrm{O}$ item TT2, indicado na tabela de medida (consultar Apêndice A), foi excluído dos resultados por apresentar um "Outer loading" embora superior a 0.40, mas inferior a 0.70. A decisão deriva do facto da análise ao impacto da exclusão do indicador demonstrar um aumento das medidas acima do limite para AVE assim como para a Confiabilidade Composta (Hair et al., 2014). É confirmada a validade discriminante através da verificação das Cargas Transversais "Cross Loadings" dos itens, sendo que os valores das cargas externas dos indicadores associados às dimensões são superiores a todos os valores de carga das restantes dimensões (Hair et al., 2014). Foi também avaliada a validade discriminante, através da aplicação do critério de Fornell-Larcker, verificado na Tabela 4, como abordagem mais conservadora para avaliar a validade discriminante, onde apenas as dimensões reflexivas são possíveis de avaliar com a aplicação do critério de Fornell-Larcker (Hair et al., 2014). 


\begin{tabular}{lllllll}
\hline & AVE & $\begin{array}{l}\text { Confiabilidade } \\
\text { composta }\end{array}$ & $\mathbf{R}^{2}$ & $\begin{array}{l}\text { Cronbachs } \\
\text { Alpha }\end{array}$ & Concordância & Redundância \\
\hline$T T$ & 0.878 & 0.935 & 0.247 & 0.861 & 0.878 & 0.217 \\
\hline$F U P$ & 0.904 & 0.966 & 0.665 & 0.947 & 0.904 & 0.600 \\
\hline$U P$ & 0.882 & 0.957 & 0.641 & 0.934 & 0.882 & 0.563 \\
\hline$B I$ & 0.918 & 0.971 & 0.657 & 0.955 & 0.918 & 0.291 \\
\hline$U S E$ & 0.773 & 0.911 & 0.468 & 0.853 & 0.773 & 0.360 \\
\hline$B a$ & 0.572 & 0.914 & 0 & 0.892 & 0.572 & 0 \\
\hline$F C O$ & 0.884 & 0.938 & 0.667 & 0.869 & 0.884 & 0.588 \\
\hline$T S$ & 0.917 & 0.957 & 0.528 & 0.910 & 0.917 & 0.484 \\
\hline & 0.710 & 0.907 & 0.856 & 0.863 & 0.710 & 0.606 \\
\hline
\end{tabular}

Tabela 3 - Critérios de Qualidade - Smart PLS v2.o

\begin{tabular}{llllllllll}
\hline & TT & FUP & UP & BI & USE & Ba* $^{*}$ & FCO & IS & TKMI \\
\hline TT & $\mathbf{0 . 9 3 7}$ & 0 & 0 & 0 & 0 & 0 & 0 & 0 & 0 \\
FUP & 0.815 & $\mathbf{0 . 9 5 1}$ & 0 & 0 & 0 & 0 & 0 & 0 & 0 \\
UP & 0.754 & 0.801 & $\mathbf{0 . 9 3 9}$ & 0 & 0 & 0 & 0 & 0 & 0 \\
BI & 0.674 & 0.732 & 0.795 & $\mathbf{0 . 9 5 8}$ & 0 & 0 & 0 & 0 & 0 \\
USE & 0.612 & 0.613 & 0.697 & 0.684 & $\mathbf{0 . 8 7 9}$ & 0 & 0 & 0 & 0 \\
Ba* & 0.497 & 0.431 & 0.422 & 0.343 & 0.362 & $\mathbf{0 . 7 5 6}$ & 0 & 0 & 0 \\
FCO & 0.406 & 0.353 & 0.342 & 0.293 & 0.259 & 0.817 & $\mathbf{0 . 9 4 0}$ & 0 & 0 \\
IS & 0.305 & 0.260 & 0.275 & 0.242 & 0.305 & 0.727 & 0.426 & $\mathbf{0 . 9 5 8}$ & 0 \\
TKMI & 0.490 & 0.427 & 0.409 & 0.313 & 0.334 & 0.925 & 0.651 & 0.517 & $\mathbf{0 . 8 4 3}$ \\
\hline
\end{tabular}

$\boldsymbol{B a}^{*}$ dimensão refletivo-refletivo (segunda ordem)

Tabela 4 - Validade Discriminante Critério de Fornell-Larcker

Com a validação do modelo estrutural, procedemos à avaliação dos caminhos estruturais para testar as hipóteses apresentadas neste estudo. Desta forma, o ambiente propício à partilha do conhecimento " $B a$ ", enquanto dimensão de segunda ordem refletivo-refletivo, porque " $B a$ " reflete-se nas dimensões FCO $(\beta=0.817, \mathrm{p}<0.001)$, IS ( $\beta=0.727, p<0.001)$ e TKMI ( $\beta=0.925, p<0.001)$, explicando em $66.7 \%, 52.8 \%$ e $85.6 \%$ respetivamente, confirmando assim a hipótese H7. Já “ $B a$ ” ( $\beta=0.497, \mathrm{p}<0.001)$ explica apenas em $24.7 \%$ da variação exercida sobre a TT, confirmando assim a hipótese H6. A dimensão referente à TT $(\beta=0.815, \mathrm{p}<0.001)$ explica em $66.5 \%$ da variação sobre FUP, confirmando assim a hipótese H1. Na Tabela 5 podemos observar os efeitos para FUP, sobre UP e BI, sendo significativo de FUP para BI e fortemente significativo de UP para BI, como seria esperado por já ter sido comprovado em diversos estudos (Venkatesh \& Davis, 2000), onde a dimensão FUP $(\beta=0.801, \mathrm{p}<0.001)$ explica em $64.1 \%$ da UP, confirmando também a hipótese H2. 


\begin{tabular}{|c|c|c|c|c|c|c|c|c|}
\hline \multirow{2}{*}{$\begin{array}{l}\text { Hipótese } \\
H 1 \\
\end{array}$} & \multicolumn{2}{|l|}{$\begin{array}{l}\text { Variável } \\
\text { Independente }\end{array}$} & \multirow{2}{*}{$\begin{array}{l}\text { Variável } \\
\text { Dependente } \\
\text { FUP } \\
\end{array}$} & \multirow{2}{*}{$\begin{array}{l}\boldsymbol{\beta} \\
0.815\end{array}$} & \multirow{2}{*}{$\begin{array}{l}\text { T-Value } \\
28.614 \\
\end{array}$} & \multicolumn{2}{|c|}{$\begin{array}{l}\text { Significância } \\
\text { pValue } \mathrm{f}^{2}\end{array}$} & \multirow{2}{*}{$\begin{array}{l}\begin{array}{l}\text { Efeito da } \\
\text { Dimensão }\end{array} \\
\text { Forte }\end{array}$} \\
\hline & $\mathrm{TT}$ & $\rightarrow$ & & & & 0.000 & 1.985 & \\
\hline $\mathrm{H}_{2}$ & FUP & $\rightarrow$ & UP & 0.801 & 23.102 & 0.000 & 1.786 & Forte \\
\hline $\mathrm{H}_{3}$ & FUP & $\rightarrow$ & $\mathrm{BI}$ & 0.266 & 3.121 & 0.002 & 0.356 & Forte \\
\hline $\mathrm{H}_{4}$ & UP & $\rightarrow$ & $\mathrm{BI}$ & 0.582 & $7 \cdot 330$ & 0.000 & 0.076 & Fraco \\
\hline$H_{5}$ & $\mathrm{BI}$ & $\rightarrow$ & USE & 0.684 & 13.898 & 0.000 & 0.880 & Forte \\
\hline \multirow[t]{2}{*}{ H6 } & $\mathrm{Ba}$ & $\rightarrow$ & $\mathrm{TT}$ & 0.497 & 8.214 & 0.000 & 0.328 & Moderado \\
\hline & & & $\mathrm{FCO}$ & 0.817 & 29.82 & 0.000 & - & \\
\hline \multirow[t]{2}{*}{$\mathrm{H}_{7}$} & $\mathrm{Ba}$ & $\rightarrow$ & IS & 0.727 & 18.088 & 0.000 & - & \\
\hline & & & TKMI & 0.925 & 103.748 & 0.000 & - & \\
\hline
\end{tabular}

NS=Não Significativo; Significativo $a^{*} p<.05$; Significativo $a{ }^{* *} p<.01$; Significativo $a * * *<.001$; Efeito Dimensão ( $\left.f^{2}\right)$ : >o.35 Forte; >0.15 e $\leq 0.35$ Moderado; >0.02 e $\leq 0.15$ Fraco (Cohen, 1992)

Tabela 5 - Resultado das Hipóteses

BI é explicada em 65.7\% pelas dimensões FUP $(\beta=0.266, p<0.01)$ e UP $(\beta=0.582$, $\mathrm{p}<0.001)$, para as hipóteses $\mathrm{H} 3$ e H4. A dimensão $\mathrm{BI}(\beta=0.684, \mathrm{p}<0.001)$ explica por sua vez $46.8 \%$ da variação exercida sobre USE, confirmando assim a hipótese $\mathrm{H}_{5}$, embora de forma moderada. O modelo estrutural KMS, apresentado na Figura 2, suporta assim todas as ligações (Cohen, 1992), como visível na demonstração gráfica do modelo estrutural KMS.

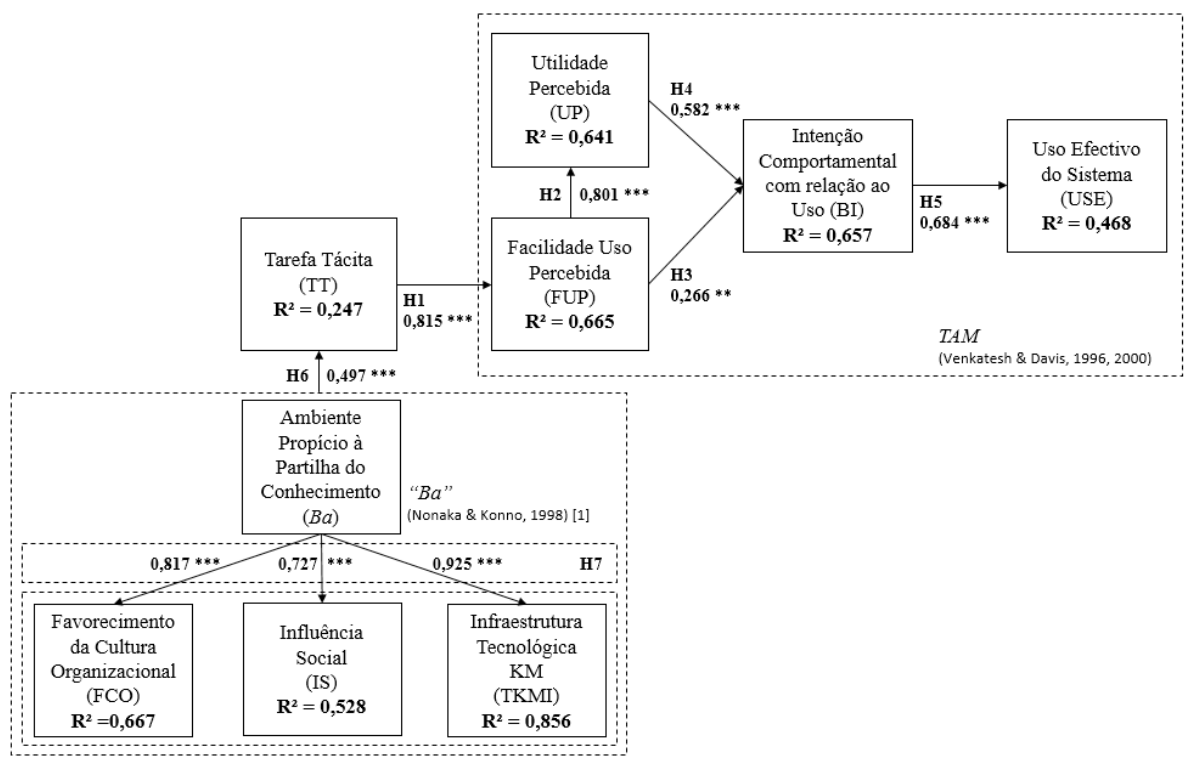

Figura 2 - Resultados - Modelo Estrutural KMS - "fonte própria” 


\subsection{Discussão}

O efeito positivo da IS é o resultado de que o uso das tecnologias CMS, alvo do estudo KMS, é considerado necessário no ponto de vista das pessoas que são importantes para nós. No entanto, no modelo estrutural KMS, " $B a$ " explica em $52.8 \%$ da variância da IS ( $\beta=0.727$, $\mathrm{p}<0.001$ ), demonstrando-se um valor bastante moderado na vertente social, que pode influenciar o ambiente de partilha de conhecimento. Este valor pode explicar em parte um efeito baixo exercido por " $B a$ " sobre a TT do colaborador, enquanto utilizador das plataformas tecnológicas KMS na organização, onde a dimensão do ambiente propício à partilha de conhecimento (“ $B a$ ”) ( $\beta=0.497, \mathrm{p}<0.001)$ apenas explica $24.7 \%$ da variação exercida sobre a TT. Segundo Nonaka \& Nishiguchi (2001), alterações drásticas, como as que envolvem a transformação de uma organização, podem afetar negativamente as relações sociais, pondo em risco a criação de conhecimento dentro das organizações, sendo uma eventual explicação sobre o valor mediano obtido para IS. Com a evolução dos SI integrados, a subsistência dos sistemas KMS pode passar pela implementação de módulos aplicacionais de redes sociais que permitam criar ou reforçar os laços de confiança para a realização da partilha de conhecimento, como componente de socialização entre colaboradores na organização e stakeholders (Nonaka \& Takeuchi, 1995; Rodriguez \& Edwards, 2008; Sousa, Costa, \& Aparicio, 2013). A tarefa tácita determina a acumulação implícita de habilidades, resultado da aprendizagem prática, onde a codificabilidade e a observabilidade são as variantes utilizadas como forma de medir o grau exercido pelo conhecimento tácito na execução de uma tarefa num determinado sistema (Kankanhalli et al., 2005). São observados valores $R^{2}$ superiores a 0.64 quando a TT $(\beta=0.815$, p<0.001) explica em $66.5 \%$ da variação sobre a FUP, demonstrando que a tarefa tácita exercida pelo utilizador assume um efeito positivo quando do uso dos sistemas KMS, sendo relevante codificar e observar a informação técnica inserida nos sistemas KMS da organização (Hair et al., 2014). Na análise efetuada aos resultados, foi verificado que os valores das dimensões FUP e UP corroboram BI, com valores $\mathrm{R}^{2}$ superiores a o.64, com níveis de significância que permitem suportar as hipóteses H2, H3 e H4 (Hair et al., 2014). Contudo, o USE em relação aos sistemas KMS avaliados neste estudo, apresenta um valor $\mathrm{R}^{2}$ moderado, explicado por BI em $46.8 \%$. Pode eventualmente, o valor moderado apresentado na hipótese $\mathrm{H}_{5}$, ser em parte explicado pelo uso da internet social como alternativa ou complemento aos sistemas internos de KMS da organização, embora o uso de soluções sociais na internet possa representar uma falha de controlo da informação obtida e disponibilizada, e consequentemente um risco para a organização (McLoughlin \& Lee, 2007; Molly McLure Wasko \& Faraj, 2005). No entanto, estas ferramentas sociais Web 2.0, categorizadas como CMS, tais como os Blogs, Wikis e redes sociais especializadas, são também consideradas ferramentas pedagógicas e de suporte técnico, utilizadas por diferentes organizações no mundo inteiro, promovendo as condições ideais para a realização da partilha da informação e de conhecimento, através da partilha de experiencias entre diferentes utilizadores (McLoughlin \& Lee, 2007). Outra explicação para o valor moderado apresentado na hipótese $\mathrm{H} 5$, pode passar pelo uso de vários sistemas KMS, neste caso específico dos sistemas CMS, poder ser contra procedente, desmotivando o utilizador final e promovendo por vezes a redundância de informação, que nem sempre representa necessariamente eficiência, já que pode resultar numa rede caótica e impraticável de informação (Stenmark, 2004). Para além disso, o uso dos sistemas CMS pode estar condicionado a diferentes aspetos relacionados com 
a perceção que cada utilizador desenvolve em relação ao uso efetivo desses sistemas, onde para alguns utilizadores o uso dos sistemas CMS está intrinsecamente associado ao desempenho imediato do seu trabalho, implicando assim um uso mais frequente da tecnologia com um objetivo mais tático, já para outros utilizadores a motivação para o uso dos sistemas CMS tem em consideração o usufruto a longo prazo, sendo intrinsecamente associado ao seu desenvolvimento pessoal, podendo assim representar uma frequência menor, com um objetivo mais estratégico (Malhotra \& Galletta, 1999).

\section{Conclusões}

Este estudo apresenta uma revisão de literatura no âmbito da gestão do conhecimento, nomeadamente no que concerne aos fatores apresentados por vários estudos, como sendo fatores influenciadores da partilha de conhecimento em ambiente organizacional. Com base na literatura, é aqui apresentado e validado um modelo estrutural de adoção de sistemas de gestão do conhecimento (KMS). O modelo explica 47\% do uso de KMS em contexto organizacional. $\mathrm{O}$ artigo contribui para o melhor entendimento dos fatores que levam à adoção de KMS, particularmente por efeito de um ambiente propício a partilha de conhecimento, concluindo-se que " $B a$ " é uma dimensão de segunda ordem que se reflete na cultura organizacional, na influencia social e na infraestrutura tecnológica. " $\mathrm{Ba}$ " influencia a tarefa tacita, que por sua vez influencia a facilidade de uso percebida. A facilidade de uso percebida e a utilidade percebida são fatores determinantes na intenção de uso de KMS, explicando 66\% dessa intenção. Trabalhos futuros podem passar pela avaliação de resultados para cada um dos sistemas em separado, atendendo ao género e idade como eventuais moderadores.

\section{Referências}

Alavi, M., Kayworth, T. R., \& Leidner, D. E. (2005). An empirical examination of the influence of organizational culture on knowledge management practices. Journal of Management Information Systems, 22(3), 191-224.

Aparicio, M., Bacao, F., \& Oliveira, T. (2016). Cultural impacts on e-learning systems' success. The Internet and Higher Education, 31, 58-70. https://doi.org/10.1016/j. iheduc.2016.06.003

Atlassian Software Systems. (2012). Jira (Version 5.0.5). Atlassian.

Atlassian Software Systems Pty Ltd. (2013). Confluence (Version 4.3.7). Retrieved from http//confluence.atlassian.com

Castellani, M. R., Reinhard, N., \&Zwicker, R. (1998). Cultura Organizacional e Tecnologia da Informação: um estudo do uso da Internet na atividade acadêmica de pesquisa. Anais do XXII Encontro da Associação Nacional de Pós-Graduação e Pesquisa em Administração, Foz do Iguaçu, Brasil.

Chaffey, D., \& Wood, S. (2005). Business Information Management: Improving Performance Using Information Systems (1st ed.). Upper Saddle River, NJ: Pearson Education 
Cohen, J. (1992). A power primer. Psychological Bulletin, 112(1), 155.

Costa, C. J., Ferreira, E., Bento, F., \& Aparicio, M. (2016). Enterprise resource planning adoption and satisfaction determinants. Computers in Human Behavior, 63, 659671. https://doi.org/10.1016/j.chb.2016.05.090

Davenport, T. H., De Long, D. W., \& Beers, M. C. (1998). Successful knowledge management projects. MIT Sloan Management Review, 39(2), 43.

Davenport, T. H., \& Prusak, L. (1998). Working knowledge: How organizations manage what they know. Brighton, MA: Harvard Business Press.

Davis, F. D., Bagozzi, R. P., \& Warshaw, P. R. (1989). User acceptance of computer technology: a comparison of two theoretical models. Management Science, 35(8), 982-1003.

Davis, F. D., \& Venkatesh, V. (1996). A critical assessment of potential measurement biases in the technology acceptance model: three experiments. International Journal of Human-Computer Studies, 45(1), 19-45.

Davis Jr, F. D. (1986). A technology acceptance model for empirically testing new enduser information systems: Theory and results. Cambridge, MA: Massachusetts Institute of Technology.

Doll, W. J., \& Torkzadeh, G. (1988). The measurement of end-user computing satisfaction. MIS Quarterly, 259-274.

Drucker, P. F. (1988). The coming of the new organization. Harvard Business Review. Retrieved from https://hbr.org/1988/o1/the-coming-of-the-new-organization

Fishbein, M., \& Ajzen, I. (1975). Belief. Attitude, Intention and Behavior: An Introduction to Theory and Research. Reading, MA: Addison-Wesley.

Fleury, M. T. L. (1997). O ambiente para aprendizagem organizacional. Educação E Empregabilidade: Novos Caminhos Da Aprendizagem. São Paulo: Educ.

Galliers, R. D., \& Leidner, D. E. (2014). Strategic information management: challenges and strategies in managing information systems. Abingdon: Routledge.

Gold, A. H., Malhotra, A., \& Segars, A. H. (2001). Knowledge management: An organizational capabilities perspective. Journal of Management Information Systems, 18(1), 185-214.

Gomes, J., \& Romão, M. (2012). Seleção de uma abordagem de gestão de investimentos em Sistemas e Tecnologias da Informação. RISTI - Revista Ibérica de Sistemas E Tecnologias de Informação, (10), 35-49.

Goodhue, D. L., \& Thompson, R. L. (1995). Task-technology fit and individual performance. MIS Quarterly, 213-236.

Goodman, P. S., \& Darr, E. D. (1998). Computer-aided systems and communities: Mechanisms for organizational learning in distributed environments. Mis Quarterly, 417-440. 
Hair Jr, J. F., Hult, G. T. M., Ringle, C., \& Sarstedt, M. (2014). A primer on partial least squares structural equation modeling (PLS-SEM). Thousand Oaks, CA: Sage Publications.

Hall, H. (2001). Input-friendliness: motivating knowledge sharing across intranets. Journal of Information Science, 27(3), 139-146.

Helfat, C. E., \& Peteraf, M. A. (2003). The dynamic resource-based view: Capability lifecycles. Strategic Management Journal, 24(10), 997-1010.

Jarvenpaa, S. L., \& Staples, D. S. (2000). The use of collaborative electronic media for information sharing: an exploratory study of determinants. The Journal of Strategic Information Systems, 9(2), 129-154.

Junnarkar, B., \& Brown, C. V. (1997). Re-assessing the enabling role of information technology in KM. Journal of Knowledge Management, 1(2), 142-148.

Kankanhalli, A., Tan, B. C., \& Wei, K. (2005). Understanding seeking from electronic knowledge repositories: An empirical study. Journal of the American Society for Information Science and Technology, 56(11), 1156-1166.

Leonard, D., \& Sensiper, S. (1998). The role of tacit knowledge in group innovation. California Management Review, 4O(3), 112-132.

Lin, H.-F. (2007). Knowledge sharing and firm innovation capability: an empirical study. International Journal of Manpower, 28(1), 315-332.

Lousã, M., \& Sarmento, A. (2016). Implementação e Utilização de Sistemas Workflow como suporte à Gestão do Conhecimento: Um estudo de caso (Vol. 3). In Atas da Conferência da Associação Portuguesa de Sistemas de Informação. Porto: APSI, ISCAP/IPP

Maier, R. (2007). Knowledge Management Systems: Information and Communication Technologies for Knowledge Management. Berlin: Springer.

Maier, R., \& Hädrich, T. (2011). Encyclopedia of Knowledge Management: Knowledge Management Systems. (David Schwartz). Idea Group Reference.

Malhotra, Y., \& Galletta, D. F. (1999). Extending the technology acceptance model to account for social influence: Theoretical bases and empirical validation. In Proceedings of the 32nd annual Hawaii international conference on Systems sciences (HICSS-32). (pp. 14). IEEE.

Markus, M. L., \& Keil, M. (1994). If we build it, they will come: Designing information systems that people want to use. Sloan Management Review, 35(4), 11.

Martins, J. M. (2010). Gestão do Conhecimento: Criação e Transferência de Conhecimento. Lisboa: Edições Sílabo.

McLoughlin, C., \& Lee, M. J. (2007). Social software and participatory learning: Pedagogical choices with technology affordances in the Web 2.0 era (pp. 664-675). In Proceedings of the ICT: Providing choices for learners and learning (ascilite 2007). Singapore 
Microsoft Coorporation. (2010). SharePoint. Retrieved from http://www.microsoft. com/sharepoint/default.mspx

Newell, S., Robertson, M., Scarbrough, H., \& Swan, J. (2009). Managing knowledge work and innovation. Basingstoke: Palgrave Macmillan.

Nonaka, I. (1994). A dynamic theory of organizational knowledge creation. Organization Science, 5(1), 14-37.

Nonaka, I., \& Konno, N. (1998). The concept of "ba": Building a foundation for knowledge creation. California Management Review, 4O(3), 40-54.

Nonaka, I., \& Nishiguchi, T. (2001). Knowledge emergence: Social, technical, and evolutionary dimensions of knowledge creation. Oxford: Oxford University Press.

Nonaka, I., \& Takeuchi, H. (1995). The knowledge-creating company: How Japanese companies create the dynamics of innovation. Oxford: Oxford University Press.

Nonaka, I., Toyama, R., \& Konno, N. (2000). SECI, Ba and leadership: a unified model of dynamic knowledge creation. Long Range Planning, 33(1), 5-34.

Noy, N. F., \& McGuinness, D. L. (2001). Ontology development 101: A guide to creating your first ontology. Retrieved from http://protege.stanford.edu/publications/ ontology_development/ontology101.pdf

Pedrosa, I., Costa, C. J., \& Laureano, R. M. (2015). Use of information technology on statutory auditors' work: New profiles beyond Spreadsheets' users (pp. 1-6). In Proceedings of the 10th Iberian Conference on Information Systems and Technologies (CISTI 2015), IEEE.

Polanyi, M. (1958). Personal knowledge, towards a post critical epistemology. Chicago, IL: University of Chicago.

Rodriguez, E., \& Edwards, J. S. (2008). Before and after modeling: Risk knowledge management is required. The Society of Actuaries.

Rosenberg, M. (2000). The e-learning readiness survey: 20 key strategic questions you and your organization must answer about the sustainability of your e-learning efforts. Retrieved July, 25, 2005 from http://books.mcgraw-hill.com/training/ elearning/elearning_survey.pdf.

Rubenstein, A. H., \& Geisler, E. (2003). Installing and managing workable knowledge management systems. Westport, CT: Greenwood Publishing Group.

Ruppel, C. P., \& Harrington, S. J. (2000). The relationship of communication, ethical work climate, and trust to commitment and innovation. Journal of Business Ethics, 25(4), 313-328.

Ryle, G. (2009). The concept of mind. Abingdon: Routledge.

Schütt, P. (2003). The post-Nonaka knowledge management. J. UCS, 9(6), 451-462.

Sousa, N., Costa, C. J., \& Aparicio, M. (2013). IO-SECI: a conceptual model for knowledge management. In Proceedings of the Workshop on Open Source and Design of Communication (pp. 9-17). ACM. 
Stenmark, D. (2004). Intranets and organisational culture. In Proceedings of IRIS 27. (pp. 14-17). Falkenberg, Sweden

Teece, D. J., Pisano, G., \& Shuen, A. (1997). Dynamic capabilities and strategic management. Strategic Management Journal, 509-533.

Tyan, K.-J. (2004). Diffusion barriers to e-learning in corporate Taiwan: A factor analysis of practitioners'perspectives. Ph. D. Dissertation thesis. Indiana University

Van Baalen, P., Bloemhof-Ruwaard, J., \& Van Heck, E. (2005). Knowledge Sharing in an Emerging Network of Practice:: The Role of a Knowledge Portal. European Management Journal, 23(3), 300-314.

Van Dam, N., \& Van Dam, N. (2004). The e-learning fieldbook: implementation lessons and case studies from companies that are making e-learning work (Vol. 360). New York, NY: McGraw-Hill.

van den Hooff, B., Vijvers, J., \& De Ridder, J. (2003). Foundations and applications of a knowledge management scan. European Management Journal, 21(2), 237-246.

Vasconcellos, L., \& Fleury, M. T. L. (2008). O desafio da adoção do e-learning pelos colaboradores de uma empresa de telecomunicações. REGE Revista de Gestão, 15(spe), 31-46.

Venkatesh, V., \& Davis, F. D. (2000). A theoretical extension of the technology acceptance model: Four longitudinal field studies. Management Science, 46(2), 186-204.

Venkatesh, V., Davis, F. D., \& Morris, M. G. (2007). Dead or alive? The development, trajectory and future of technology adoption research. Journal of the Association for Information Systems, 8(4), 267.

Venkatesh, V., Morris, M. G., Davis, G. B., \& Davis, F. D. (2003). User acceptance of information technology: Toward a unified view. MIS Quarterly, 425-478.

Wasko, M. M., \& Faraj, S. (200o). "It is what one does": why people participate and help others in electronic communities of practice. The Journal of Strategic Information Systems, 9(2), 155-173.

Wasko, M. M., \& Faraj, S. (2005). Why should I share? Examining social capital and knowledge contribution in electronic networks of practice. MIS Quarterly, 35-57.

Wilson, T. D. (2002). The nonsense of knowledge management. Information Research, $8(1), 8-1$.

Xavier, L. A., Oliveira, M., \& Teixeira, E. K. (2012). Teorias utilizadas nas investigações sobre gestão do conhecimento. RISTI - Revista Ibérica de Sistemas e Tecnologias de Informação, (10), 1-17. 


\section{Apêndice A}

\begin{tabular}{|c|c|c|c|}
\hline Dimensão & Cód. Item & Item & Referências \\
\hline \multirow{3}{*}{$\begin{array}{l}\text { Utilidade } \\
\text { Percebida } \\
\text { (Perceived usefulness) }\end{array}$} & UP1 & $\begin{array}{l}\text { Utilizar o sistema } \\
\text { minha produtividade. }\end{array}$ & \multirow{3}{*}{$\begin{array}{l}\text { Davis et al. } \\
1989\end{array}$} \\
\hline & UP2 & $\begin{array}{l}\text { Eu acho que o sistema ___ é útil para o } \\
\text { meu trabalho. }\end{array}$ & \\
\hline & UP3 & $\begin{array}{l}\text { Usar o sistema } \subsetneq \text { no meu trabalho } \\
\text { permite-me realizar tarefas de forma mais } \\
\text { célere. }\end{array}$ & \\
\hline \multirow{3}{*}{$\begin{array}{l}\text { Facilidade de Uso Percebida } \\
\text { (Perceived ease of use) }\end{array}$} & FUP1 & $\begin{array}{l}\text { A minha interação com o sistema __ é } \\
\text { clara e compreensível. }\end{array}$ & \multirow{3}{*}{$\begin{array}{l}\text { Davis et al., } \\
1989\end{array}$} \\
\hline & FUP2 & $\begin{array}{l}\text { Acho que é fácil aceder ao sistema } \\
\text { para fazer o que pretendo fazer. }\end{array}$ & \\
\hline & FUP3 & $\begin{array}{l}\text { Eu entendo que o sistema ___ é fácil de } \\
\text { utilizar. }\end{array}$ & \\
\hline \multirow{3}{*}{$\begin{array}{l}\text { Atitude para com a } \\
\text { utilização do Sistema } \\
\text { (Attitude towards system } \\
\text { use) }\end{array}$} & - & Utilizar o sistema ___ é uma má/boa ideia. & \multirow{3}{*}{$\begin{array}{l}\text { Davis et al., } \\
1989\end{array}$} \\
\hline & - & $\begin{array}{l}\text { Eu não gosto/gosto da ideia de utilizar o } \\
\text { sistema }\end{array}$ & \\
\hline & - & $\begin{array}{l}\text { Usar o sistema __ é desagradável / } \\
\text { agradável. }\end{array}$ & \\
\hline \multirow{3}{*}{$\begin{array}{l}\text { Intenção Comportamental } \\
\text { de Uso do Sistema } \\
\text { (Behavioral intention to } \\
\text { use) }\end{array}$} & BI1 & $\begin{array}{l}\text { Assumindo que tenho acesso ao sistema } \\
\text {, intenciono utiliza-lo. }\end{array}$ & \multirow{3}{*}{$\begin{array}{l}\text { Davis et al., } \\
1989\end{array}$} \\
\hline & BI2 & $\begin{array}{l}\text { Eu intenciono utilizar o sistema __ nos } \\
\text { próximos } 12 \text { meses. }\end{array}$ & \\
\hline & BI3 & Eu intenciono utilizar muitas vezes o sistema & \\
\hline \multirow{3}{*}{$\begin{array}{l}\text { Uso Efetivo } \\
\text { (USE) }\end{array}$} & USE1 & Eu utilizo atualmente o sistema & $\begin{array}{l}\text { Davis et al., } \\
1992\end{array}$ \\
\hline & USE2 & $\begin{array}{l}\text { Utilizo o sistema __ para obter a } \\
\text { informação de apoio no meu trabalho. }\end{array}$ & \multirow{2}{*}{$\begin{array}{l}\text { Urbach et al, } \\
2010\end{array}$} \\
\hline & USE3 & $\begin{array}{l}\text { Utilizo o sistema _ para guardar e } \\
\text { partilhar documentos e informação. }\end{array}$ & \\
\hline \multirow[b]{2}{*}{ Influência Social (IS) } & IS1 & $\begin{array}{l}\text { As pessoas, que são importantes para mim, } \\
\text { entendem que eu devo utilizar os sistemas de } \\
\text { apoio ao conhecimento. }\end{array}$ & \multirow{2}{*}{$\begin{array}{l}\text { Venkatesh and } \\
\text { Davis, } 2000\end{array}$} \\
\hline & IS2 & $\begin{array}{l}\text { As pessoas, que influenciam o meu } \\
\text { comportamento, entendem que eu } \\
\text { deveria utilizar os sistemas de apoio ao } \\
\text { conhecimento. }\end{array}$ & \\
\hline \multirow{2}{*}{$\begin{array}{l}\text { Favorecimento da } \\
\text { Cultura Organizacional } \\
\text { (FCO) }\end{array}$} & FCO1 & $\begin{array}{l}\text { A cultura da minha organização favorece } \\
\text { a adoção de sistemas de apoio ao } \\
\text { conhecimento. }\end{array}$ & \multirow{2}{*}{$\begin{array}{l}\text { Rosenberg, } \\
2000\end{array}$} \\
\hline & FCO2 & $\begin{array}{l}\text { A cultura da minha organização valoriza a } \\
\text { aprendizagem. }\end{array}$ & \\
\hline
\end{tabular}




\begin{tabular}{|c|c|c|c|c|}
\hline \multicolumn{2}{|l|}{ Dimensão } & \multirow{2}{*}{$\begin{array}{l}\text { Cód. Item } \\
\text { FI1 }\end{array}$} & \multirow{2}{*}{$\begin{array}{l}\text { Item } \\
\text { Eu gosto de ajudar os meus colegas } \\
\text { compartilhando o meu conhecimento com } \\
\text { eles. }\end{array}$} & \multirow{3}{*}{$\begin{array}{l}\text { Referências } \\
\text { Wasko } \\
\text { and Faraj, } \\
2000\end{array}$} \\
\hline \multirow{4}{*}{$\begin{array}{l}\text { Fatores } \\
\text { Individuais } \\
\text { (FI) }\end{array}$} & \multirow{2}{*}{$\begin{array}{l}\text { Enjoyment } \\
\text { in helping } \\
\text { others }\end{array}$} & & & \\
\hline & & FI2 & $\begin{array}{l}\text { É um prazer compartilhar meu } \\
\text { conhecimento com os meus colegas. }\end{array}$ & \\
\hline & \multirow{2}{*}{$\begin{array}{l}\text { Knowledge } \\
\text { self- } \\
\text { efficacy }\end{array}$} & FI3 & $\begin{array}{l}\text { Estou confiante na minha capacidade de } \\
\text { criar conhecimento que outros na minha } \\
\text { organização consideram valioso. }\end{array}$ & \multirow{2}{*}{ Spreitzer, 1995} \\
\hline & & FI4 & $\begin{array}{l}\text { Eu tenho a experiência necessária para } \\
\text { fornecer conhecimentos valiosos para a } \\
\text { minha organização. }\end{array}$ & \\
\hline \multirow{3}{*}{\multicolumn{2}{|c|}{ Tarefa Tácita (TT) }} & TT1 & $\begin{array}{l}\text { O conhecimento utilizado é codificável no } \\
\text { sistema }\end{array}$ & \multirow{3}{*}{$\begin{array}{l}\text { Subramaniam } \\
\& \\
\text { Venkatraman, } \\
2001\end{array}$} \\
\hline & & TT2 & $\begin{array}{l}\text { O conhecimento utilizado é complexo no } \\
\text { sistema }\end{array}$ & \\
\hline & & TT3 & $\begin{array}{l}\text { O conhecimento utilizado é observável no } \\
\text { sistema }\end{array}$ & \\
\hline \multirow{3}{*}{\multicolumn{2}{|c|}{$\begin{array}{l}\text { Interdependência de } \\
\text { Tarefas (IT) }\end{array}$}} & IT1 & $\begin{array}{l}\text { As tarefas no sistema ___ dependem de } \\
\text { outra unidade. }\end{array}$ & \multirow{3}{*}{$\begin{array}{l}\text { Jarvenpaa and } \\
\text { Staples, } 2000\end{array}$} \\
\hline & & IT2 & $\begin{array}{l}\text { As tarefas no sistema ___ dependem de } \\
\text { outro departamento. }\end{array}$ & \\
\hline & & IT3 & $\begin{array}{l}\text { As tarefas no sistema ___ dependem de } \\
\text { outra entidade. }\end{array}$ & \\
\hline \multirow{4}{*}{\multicolumn{2}{|c|}{$\begin{array}{l}\text { Technological KM } \\
\text { Infrastructure (TKMI) }\end{array}$}} & TKM1 & $\begin{array}{l}\text { Minha organização usa tecnologia que } \\
\text { permite aos colaboradores partilharem } \\
\text { informação e conhecimento com outras } \\
\text { pessoas dentro da organização. }\end{array}$ & \multirow{4}{*}{$\begin{array}{l}\text { Gold et al., } \\
2001\end{array}$} \\
\hline & & TKM2 & $\begin{array}{l}\text { Minha organização usa tecnologia que } \\
\text { permite que pessoas em vários locais } \\
\text { aprendam como um só grupo heterogéneo } \\
\text { ou em diversas fases no tempo. }\end{array}$ & \\
\hline & & TKM3 & $\begin{array}{l}\text { Minha organização utiliza tecnologia que } \\
\text { permite a busca de novos conhecimentos. }\end{array}$ & \\
\hline & & TKM4 & $\begin{array}{l}\text { Minha organização utiliza tecnologia que lhe } \\
\text { permite recuperar e usar o conhecimento } \\
\text { sobre seus produtos e processos. }\end{array}$ & \\
\hline
\end{tabular}

Tabela de Medida das Dimensões 